\title{
Vai trò vốn xã hội của tổ chức trong việc tăng chia sẻ kiến thức trong khu vực công tại Việt Nam
}

\section{The role of organizational social capital in increasing knowledge sharing within the public sector in Vietnam}

\author{
Nguyễn Văn Phương ${ }^{1 *}$, Nguyễn Thị Thùy Quyên ${ }^{2}$, Nguyễn Thị Thanh Ngân ${ }^{3}$ \\ ${ }^{1}$ Trường Đại học Quốc tế, Đại học Quốc gia Thành Phố Hồ Chí Minh, Việt Nam \\ ${ }^{2}$ Học viện Cán bộ Thành phố Hồ Chí Minh, Việt Nam \\ ${ }^{3}$ Trường Đại học Lao động Xã hội (Cơ sở 2) Thành phố Hồ Chí Minh, Việt Nam \\ *Tác giả liên hệ, Email: nvphuong@hcmiu.edu.vn
}

THÔNG TIN

DOI: $10.46223 / \mathrm{HCMCOUJS}$. econ.vi.16.3.1349.2021

Ngày nhận: 11/12/2020

Ngày nhận lại: 18/02/2021

Duyệt đăng: 23/02/2021

\section{Tì khóa:}

chia sẻ kiến thức; động lực phụng sự; thái độ; vốn xã hội của tổ chức

Keywords:

knowledge sharing; public service motivation; attitude; organizational social capital

\section{TÓM TẮT}

Nghiên cứu này hướng đến thiết lập mô hình nghiên cứu về chia sẻ kiến thức trong khu vực công và làm rõ vai trò vốn xã hội của tổ chức trong khu vực công. Mô hình chia sẻ kiến thức được phát triển trên cơ sở xác định mối tương quan đa chiều của vốn xã hội của tổ chức với thái độ và động lực phụng sự, từ đó tác động đến việc gia tăng chia sẻ kiến thức. Nghiên cứu này dựa trên khảo sát của 319 nhà quản lý hiện đang công tác tại các đơn vị hành chính sự nghiệp và các tổ chức xã hội thuộc khu vực công và sử dụng mô hình cấu trúc để kiểm định giả thuyết. Kết quả cho thấy thái độ và vốn xã hội của tổ chức có ảnh hưởng to lớn đến việc chia sẻ kiến thức. Trong khi đó, không có bằng chứng củng cố cho sự ảnh hưởng trực tiếp của động lực phụng sự lên quá trình chia sẻ kiến thức. Nghiên cứu cũng chỉ ra những đóng góp về mặt lý thuyết và đưa ra những đề xuất cụ thể để thúc đẩy công chức tham gia vào các hoạt động chia sẻ kiến thức.

ABSTRACT
This study aims to establish a research model of knowledge
sharing in the public sector and attempts to clarify the role of
organizational social capital in the public sector. The knowledge-
sharing model is developed based on determining the
multidimensional relationship of the organizational social capital
with attitudes and public service motivation, thereby influencing
the increase in knowledge-sharing. The research is based on a
survey of 319 managers working in public organizations and non-
business units and social organizations in the public sector and
using a structural equation model to test hypotheses. The results
show that attitude and organizational social capital have a
significant influence on knowledge sharing. Meanwhile, there is
no evidence to support the direct effect of public service
motivation on the knowledge-sharing process. The research also
clarifies the theoretical contributions and gives specific
recommendations to motivate civil servants to participate in
knowledge-sharing activities.




\section{Giới thiệu}

Khái niệm về kiến thức được hiểu là thông tin được xử lý bởi các cá nhân, gồm những ý tưởng, kỹ năng và đánh giá phù hợp với hiệu suất của cá nhân, nhóm và tổ chức (Alavi \& Leidner, 2001; Bartol \& Srivastava, 2002). Chia sẻ kiến thức được đánh giá có vai trò vô cùng quan trọng trong sự thành công của một tổ chức, đặc biệt là về khía cạnh tài chính và kinh tế, Theo Wang, Noe, và Wang (2014) kiến thức đóng vai trò quan trọng trong nguồn lực của một tổ chức, kiến thức là một nguồn tài nguyên về lợi thế cạnh tranh giữa các công ty. Và chia sẻ kiến thức sẽ đóng góp trong áp dụng những kiến thức và tạo ra những tri thức mới và sau cùng đó chính là lợi thế cạnh tranh cho tổ chức (Jackson, Chuang, Harden, \& Jiang, 2006). Việc chủ động chia sẻ kiến thức giúp doanh nghiệp nắm bắt được cơ hội thành công bằng cách "tạo điều kiện thuận lợi cho việc ra quyết định, xây dựng văn hóa học tập thông qua việc tạo thói quen học tập thường xuyên, và kích thích thay đổi, cải tiến về mặt văn hóa." Do vậy, thấu hiểu được việc chia sẻ kiến thức và các yếu tố ảnh hưởng của nó sẽ tạo ra được đóng góp đáng kể cho việc tìm ra được nguyên nhân và nguồn gốc của sự thành công lâu dài. Các tổ chức khu vực công là các tổ chức phi lợi nhuận bao gồm các cơ quan chính phủ, các tập đoàn và các cơ quan quân sự có nhiệm vụ và thực hiện các dịch vụ công. Đối với tổ chức phi lợi nhuận như các tổ chức khu vực công, "chia sẻ kiến thức là cách thức để tăng năng suất và cải thiện sự hài lòng của người dân và nhân viên” (Pan \& Scarbrough, 1998).

Mặc dù thuật ngữ "kiến thức" được sử dụng từ lâu và khá thường xuyên, nhưng vấn đề về quản trị kiến thức và chia sẻ kiến thức mới được nghiên cứu sâu từ những năm 1990. Trong một cuốn sách vô cùng phổ biến "Working knowledge: How organizations manage what they know”, Prusak và Davenport (1998) nhấn mạnh vai trò của kiến thức trong một tổ chức nhằm giúp trả lời câu hỏi tại sao một số công ty lại liên tục thành công. Trong cuốn sách này, các nhà quản lý khôn ngoan từ 25 công ty (bao gồm Hewlett Packard, IBM, AT\&T, và American Airlines) thừa nhận rằng "bản thân họ không có bất kì biện pháp hay phương thức tiếp cận hiệu quả nào đối với việc quản lý hay hiểu được cách sử dụng thông tin tốt hơn, nói cách khác là việc sử dụng kiến thức hiệu quả." Có chung nỗi lo này, trong những năm gần đây các nhà nghiên cứu và học giả đã đặt vấn đề về sự cần thiết tạo ra văn hóa tổ chức cởi mở, giao tiếp tự do, chia sẻ kiến thức và áp dụng nhiều chiến lược đa dạng hơn. Cùng lúc đó, các tổ chức trên thế giới nói chung và các tổ chức ngân hàng đã và đang nỗ lực giới thiệu những kỹ năng quản trị kiến thức hiệu quả bằng cách khuyến khích nhân viên cải thiện hành vi chia sẻ kiến thức trong công việc hằng ngày để trí thông minh của họ được sử dụng rộng rãi, đạt được hiệu suất làm việc tốt, giúp tổ chức tăng trưởng nhanh và bền vững. Nhưng làm sao để cải thiện hành vi chia sẻ kiến thức? Những yếu tố nào ảnh hưởng đến việc chia sẻ kiến thức này?

Hành vi chia sẻ kiến thức được xem xét và kết luận là chịu ảnh hưởng từ nhiều yếu tố trực tiếp và gián tiếp. Chen, Hsieh, và Chen (2014) chỉ ra mối quan hệ tích cực giữa việc chia sẻ kiến thức và động lực phụng sự ở khu vực công tại Đài Loan. Cùng chung một kết luận, Kim (2018) lần lượt xác nhận ảnh hưởng đáng kể của cả động lực phụng sự và vốn xã hội của tổ chức lên việc chia sẻ kiến thức trong khu vực công ở Hàn Quốc. Những phát hiện thực nghiệm này đã thúc đẩy tác giả tiến hành nghiên cứu thực nghiệm để kiểm tra mô hình nghiên cứu về chia sẻ kiến thức ở khu vực công tại Việt Nam. Việt Nam, Hàn Quốc và Đài Loan có nhiều nét tương đồng về truyền thống do chịu ảnh hưởng từ Nho giáo và xã hội nông nghiệp phong kiến, tác động đến hành vi thường ngày của người dân cho đến ngày nay (Huang \& Chang, 2017). Tuy nhiên, các điểm khác biệt về chính trị, hệ thống hành chính, tầng lớp kinh tế và tiêu chuẩn sống có thể dẫn đến khác biệt về hành vi chia sẻ, đặc biệt là đối với các tài sản vô hình như kiến thức. Thêm vào đó, có rất ít nghiên cứu về chủ đề này tại Việt Nam. Do vậy, 
việc có hay không một mối quan hệ tích cực giữa động lực phụng sự và chia sẻ kiến thức, vốn xã hội của tổ chức và chia sẻ kiến thức trong khu vực công của Việt Nam vẫn còn là một ẩn số. Chính lỗ hổng trên đã hối thúc tác giả phải xem xét nó trong nghiên cứu này nhằm tìm ra những yếu tố quyết định nổi bật trong môi trường làm việc của khu vực công nhằm nhấn mạnh vai trò của việc chia sẻ kiến thức. Bằng cách sử dụng cách tiếp cận mô hình phương trình cấu trúc (SEM) để phân tích khảo sát bảng hỏi với 319 nhà quản lý làm việc cho các đơn vị hành chính sự nghiệp và các tổ chức xã hội thuộc khu vực công tại Thành phố Hồ Chí Minh.

\section{Cơ sở lý thuyết và mô hình nghiên cứu}

\subsection{Chia sẻ kiến thức}

Kiến thức sẽ được tăng lên bằng cách chia sẻ thay vì tích trữ. Mọi người luôn truyền đạt kiến thức tích lũy và trí tuệ của mình cho các thế hệ sau bằng thông qua giao tiếp để chia sẻ về suy nghĩ, công việc và kinh nghiệm (Smith, 2001). Chia sẻ kiến thức (KS) được hiểu là sự cung cấp những thông tin liên quan đến nhiệm vụ đang thực hiện và cách thức giải quyết công việc, tạo ra những ý tưởng mới và hoàn thành các chính sách hoặc các kế hoạch (Lin, Ye, \& Bi, 2014; Wang \& Noe, 2010). Bartol và Srivastava (2002) định nghĩa KS là quy trình thông qua đó nhân viên truyền bá những kiến thức liên quan đến những người khác trong tổ chức. Đó là quá trình cùng nhau trao đổi kiến thức và tạo ra kiến thức mới; nó ngụ ý là sự hợp tác của những cá nhân làm việc với mục tiêu chung (Boland \& Tenkasi, 1995). Mục tiêu cuối cùng của KS là nỗ lực chuyển kinh nghiệm, kiến thức của từng cá nhân trở thành tài sản, nguồn lực của tổ chức, nhằm nâng cao hiệu quả làm việc chung của tổ chức (Yang \& Wan, 2004). Chia sẻ kiến thức là bước quan trọng để quản lý tri thức thành công (Lee \& Ahn, 2007; Vij \& Farooq, 2014a, 2014b).

\subsection{Vốn xã hội của tổ chức}

Thuật ngữ "vốn xã hội” được sử dụng lần đầu tiên trong bài nghiên cứu cộng đồng, với ý nghĩa là những mối quan hệ về lòng tin được đưa vào các mạng lưới xã hội (Jacobs, 1961). Sau đó, nó được phát triển bởi (Coleman, 1988), người tranh luận rằng các mối quan hệ xã hội trở thành nguồn vốn như một phương tiện cất giữ giá trị. Nhưng Leana và Buren (1999) đã phát triển thuật ngữ "vốn xã hội của tổ chức" (OSC) và định nghĩa nó là một nguồn tài nguyên phản ánh tính chất của các mối quan hệ xã hội trong tổ chức và được nhận biết “thông qua các cấp độ định hướng mục tiêu chung và lòng tin giữa các thành viên nhằm tạo ra hành động tập thể hướng đến việc tạo ra giá trị." Những định nghĩa khác của OSC tập trung vào khía cạnh "gắn kết" (Adler \& Kwon, 2002), bắt đầu từ giả định rằng các cấp độ của sự liên kết và lòng tin giữa các bên trong mạng lưới phải vững chắc nhằm đảm bảo việc thực hiện các mục tiêu chung (Leana \& Buren, 1999). Ở mức độ liên kết và lòng tin cao, vốn xã hội được tạo điều kiện của doanh nghiệp dù là gắn kết hay bắc cầu cũng cần thiết cho việc thống nhất kiến thức. Chiu, Hsu, và Wang (2006) điều tra động lực chia sẻ kiến thức ở các cộng đồng mạng dựa trên thông tin thu thập từ 310 thành viên của một cộng đồng chuyên môn trực tuyến BlueShop để nhận diện hành vi chia sẻ kiến thức. Kết quả chỉ ra rằng các khía cạnh của vốn xã hội có liên hệ tích cực đến khối lượng và chất lượng của kiến thức được chia sẻ giữa các thành viên.

Các thành viên của tổ chức được khuyến khích chia sẻ hiểu biết của mình vì các hoạt động chia sẻ kiến thức như vậy đã trở thành một phần thiết yếu để duy trì lợi thế cạnh tranh cho sự tồn tại của tổ chức. Tuy nhiên, đẩy mạnh chia sẻ kiến thức không phải là một việc đơn giản do con người thường có khuynh hướng giữ lại kiến thức, kinh nghiệm của mình như nguồn tài nguyên mật. Các sáng kiến đầu tiên về việc quản trị kiến thức tập trung vào cung cấp cơ sở dữ liệu số hóa, hệ thống mạng, và phần mềm nhằm khuyến khích việc phân bổ kiến thức (Darvish \& Nikbakhsh, 2010). Các cách tiếp cận mở rộng thì lại tập trung vào phương pháp nhận thức xã hội 
nhằm đẩy mạnh thái độ và hành vi có ích trong việc tăng cường chia sẻ kiến thức, bao gồm các yếu tố như quà tặng khích lệ, phần thưởng, lòng tin, mối quan hệ, etc. (Chow \& Chan, 2008).

\subsection{Thái độ}

Gagne và Medsker (1996) đưa ra định nghĩa thái độ là một trạng thái bên trong ảnh hưởng đến lựa chọn hành động của một cá nhân hoặc xu hướng phản ứng theo trạng thái đó. Thuyết nhận thức xã hội cũng nói rằng khi một người tin rằng một người khác sẽ hành động theo cách cần thiết để đạt được kết quả mong muốn, thiện cảm về hành động đó sẽ được hình thành (Bandura, 1989). Ngoài ra, dự định thực hiện một hành vi được quyết định bởi thái độ của người đó đối với chính hành động (Ajzen \& Fishbein, 1980). Trong khi đó, thái độ cá nhân đối với một hành vi lại được tranh luận rằng là một yếu tố dự đoán quan trọng về dự định có thực hiện hành vi đó hay không (Darvish \& Nikbakhsh, 2010). Trong một nghiên cứu khác về việc gây quỹ cộng đồng cho một giải pháp mới, Martinez (2017) xác nhận vai trò trung gian cục bộ của niềm tin hệ thống đối với động lực chủ quan và dự định chia sẻ kiến thức. $\mathrm{Hsu}, \mathrm{Ju}$, Yen, và Chang (2007) cho rằng thách thức lớn nhất về kiến thức chia sẻ là sự sẵn lòng (thái độ) của cá nhân. Đó là, thái độ tiêu cực có xu hướng giảm khả năng chia sẻ kiến thức. Cùng chung quan điểm đó (Bock, Zmud, Kim, \& Lee, 2005) xem xét việc hình thành dự định thực hiện hành vi chia sẻ kiến thức thông qua khảo sát với 154 nhà quản lý ở Hàn Quốc và phát hiện rằng thái độ của một người với việc chia sẻ kiến thức càng dễ chịu, thì họ càng có khuynh hướng thực hiện chia sẻ kiến thức hơn.

\section{4. Đọng lục phụng sụr}

Động lực phụng sự (PSM) được định nghĩa là sự sẵn lòng của mỗi cá nhân trong việc thực hiện các hành vi tự nguyện mang đến điều tốt đẹp cho mỗi người mà không mong đợi được trả ơn. PSM còn được xem là niềm tin, giá trị, thái độ hoặc ám chỉ khuynh hướng phục vụ cộng đồng và lợi ích chung của một người (Brewer \& Seiden, 1998). Mặt khác, PSM được xem như khuynh hướng phản hồi của một cá nhân trước những động lực bắt nguồn chính hoặc độc nhất từ các cơ quan nhà nước. Khái niệm này gồm có bốn phương diện: mong muốn tạo chính sách công, cam kết vì lợi ích tập thể hay trách nhiệm dân sự, tự hy sinh, và sự thỏa hiệp (Perry, 1996). Từ đây, nhiều tác giả đã áp dụng bốn phương diện của Perry để đo PSM (Coursey \& Pandey, 2007; Wright \& Pandey, 2008). Tuy nhiên, sự đo lường này đã được thay đồi và điều chỉnh cho phù hợp vì nó không phù hợp để đo lường trong một vài bối cảnh nghiên cứu (Kim, 2009; Kim \& Vandenabeele, 2010; Perry \& Vandenabeele, 2015).

Nhưng mối liên hệ giữa động lực phục vụ cộng đồng và vốn xã hội của tổ chức có thể đặc biệt phức tạp. Một nghiên cứu cá nhân chỉ ra rằng các cộng đồng (tập thể) có khuynh hướng tích cực về mặt chính trị khi các đơn vị dịch vụ công hoạt động không hiệu quả, nhưng lại có thể tập trung nhiều hơn cho các hoạt động xã hội khi họ tin rằng nhu cầu của mình được đáp ứng đầy đủ (Vigoda, 2002). Trong nghiên cứu của mình, Moynihan và Pandey (2007) đã chỉ ra mối tương quan giữa động lực phụng sự và vốn tổ chức của xã hội, động lực dịch vụ công không chỉ được hình thành bởi các yếu tố lịch sử xã hội trước khi nhân viên vào làm trong tổ chức mà còn bị ảnh hưởng bởi môi trường tổ chức nơi mà nhân viên đó đang làm việc. Trong khi đó, một vài nghiên cứu về các loại vốn xã hội phát hiện ra rằng vốn xã hội có tác động tích cực đến chất lượng dịch vụ công (Andrews, 2012). Hơn thế, nhân viên có nhiều động lực thường sẽ tích cực làm việc trong khu vực công. Họ cũng sẽ dễ tập trung vào hoặc tham gia vào các hoạt động chia sẻ kiến thức vì việc làm giúp họ cống hiến nhiều hơn cho các dịch vụ công và gia tăng lợi ích công.

Sau khi khảo lược để tìm hiểu các nghiên cứu liên quan, tác giả xây dựng mô hình nghiên cứu như Hình 1 với các giả thiết sau:

H1: Vốn xã hội của tổ chức có ảnh hưởng tích cưc với việc chia sẻ kiến thức 
H2: Vốn xã hội của tổ chức có ảnh hưởng tích cực với thái đội đối với việc chia sẻ kiến thức

H3: Thái độ đối với việc chia sẻ kiến thức có liên hệ tích cực đến việc chia sẻ kiến thức

H4: Vốn xã hội của tổ chức có tác động tích cực đến động lực phụng sự

H5: Động lục phụng sự có tác động tích cực đến việc chia sẻ kiến thức

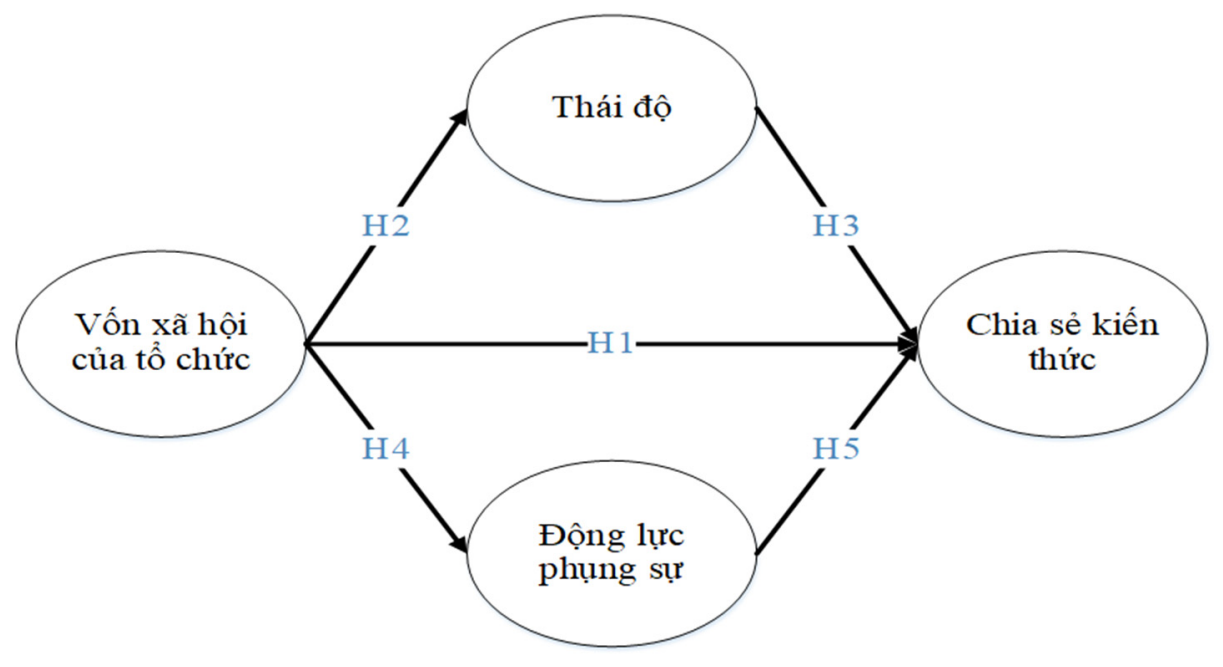

Hình 1. Mô hình nghiên cứu đề xuất

\section{Phương pháp nghiên cứu}

Đối tượng nghiên cứu là những cá nhân đang làm việc tại các cơ quan khu vực công ở Thành phố Hồ Chí Minh. Bảng hỏi khảo sát được dịch từ tiếng Anh sang tiếng Việt trước khi được phát cho người tham gia. Dựa trên phương pháp dịch ngược của (Brislin, 1970), các tác giả nhờ một biên dịch tiếng Anh tiến hành dịch ngược để đảm bảo bảng câu hỏi tiếng Anh và tiếng Việt tương tự như nhau. Các yếu tố được đo trong nghiên cứu này được ứng dụng từ các tài liệu trước đây và sửa đổi từ kết quả có được sau nhiều lần thảo luận nhóm với 12 vị lãnh đạo thuộc khu vực công cho phù hợp với bảng hỏi tiếng Việt.

Thang đo với 24 biến quan sát để đánh giá bốn biến tiềm ẩn được trình bày trong Hình 1 . Đầu tiên, Thái độ được kế thừa từ (Bock et al., 2005). Sau khi thảo luận thì thang đo cho tiêu chí này là: (1) Tôi có thái độ tích cực về việc chia sẻ kiến thức với nhân viên cấp dưới, (2) Tôi thích chia sẻ kiến thức với đồng nghiệp, (3) Tôi tin rằng việc chia sẻ kiến thức với các thành viên tại văn phòng là đáng quý, (4) Tôi thực hiện các hoạt động chia sẻ kiến thức với cấp dưới và đồng nghiệp một cách khôn ngoan.

Thứ hai, để đánh giá động lực phụng sự, một thang đo sáu biến quan sát được ứng dụng từ (Wright, Hassan, \& Park, 2016) và từ các buổi phỏng vấn với 12 vị lãnh đạo được chia thành bốn nhóm. Tuy thang đo này không cho phép chúng tôi phân biệt giữa những phương diện khác nhau của động lực phụng sự nhưng lại tập trung vào mong muốn của công - viên chức được giúp đỡ và đem lại lợi ích cho mọi người trong xã hội. Ba biến quan sát được mượn từ (Wright et al., 2016) là: (1) Tôi quan tâm sâu sắc đến việc đem lại lợi ích cho mọi người trong xã hội bằng công việc của mình, (2) Tôi cảm thấy được khuyến khích làm việc tốt để giúp đỡ mọi người trong xã hội bằng công việc của mình, (3) Tôi cảm thấy được khuyến khích làm việc tốt vì tôi muốn tạo nên ảnh hưởng tích cực đến mọi người. Những biến quan sát còn lại được đặt ra nhờ vào gợi ý của các nhà lãnh đạo tham gia thảo luận: (4) Tôi cảm thấy được khuyến khích làm việc tốt vì tôi muốn được thăng tiến trong công ty, (5) Nếu không được thăng chức trong vòng 03 đến 05 năm, tôi sẽ cảm thấy thất vọng, (6) Tôi sẽ thấy hài lòng với công việc của mình hơn nếu tôi được thăng chức. 
Thứ ba, việc đo lường vốn xã hội của tổ chức được kế thừa và phát triển từ (Kianto \& Waajakoski, 2010; Kim, 2018; Leana \& Pil, 2006). Những khái niệm chính về vốn xã hội của tổ chức bao gồm tính liên hệ và lòng tin. Các biến quan sát liên quan đến vốn xã hội của tổ chức gồm: (1) Các nhân viên trong phòng/ban của tôi có cùng tham vọng và tầm nhìn để đóng góp cho công ty, (2) Các nhân viên trong phòng/ban của tôi đều nhiệt tình theo đuổi mục tiêu và sứ mệnh tập thể, (3) Các nhân viên trong phòng/ban của tôi có một mục đích chung khi làm việc cùng nhau, (4) Các nhân viên trong phòng/ban của tôi cam kết với mục tiêu của tổ chức, (5) Các nhân viên trong phòng/ban của tôi thân ái giúp đỡ lẫn nhau, (6) Các nhân viên trong phòng/ban của tôi có niềm tin vào nhau, (7) Các nhân viên trong phòng/ban của tôi trung thực trong công việc, (8) Các nhân viên trong phòng/ban của tôi có tinh thần đồng đội.

Cuối cùng, một thang đo sáu biến quan sát được kế thừa từ (Kim, 2018) để đo việc chia sẻ kiến thức. Thang đo này gồm: (1) Tôi chủ động chia sẻ kiến thức chuyên môn với đồng nghiệp, (2) Tôi chia sẻ kinh nghiệm làm việc và kiến thức với các thành viên cùng nhóm, (3) Tôi hướng dẫn các thành viên cùng nhóm cách thực hiện phần việc khó, (4) Tôi cố gắng chia sẻ kiến thức tôi học được với các thành viên cùng nhóm hiệu quả hơn, (5) Tôi thường dành nhiều thời gian chia sẻ kiến thức với các thành viên cùng nhóm, (6) Tôi chủ động trả lời câu hỏi mà các thành viên cùng nhóm đặt ra.

Bảng 1 trình bày chi tiết các biến quan sát cụ thể cùng nguồn trích dẫn thang đo đã đuợc kế thừa và hiệu chỉnh. Tác giả sử dụng thang đo Likert 5 điểm, từ 1 là "hoàn toàn không đồng ý" đến 5 là "hoàn toàn đồng ý".

\section{Bảng 1}

Mô tả các biến và thang đo nghiên cứu

\begin{tabular}{|c|c|c|}
\hline \multicolumn{2}{|r|}{ Thang đo } & Nguồn tham khảo \\
\hline \multicolumn{3}{|c|}{ Thái độ (ATT) } \\
\hline 1 & $\begin{array}{l}\text { Tôi chia sẻ kiến thức công việc một cách vui vẻ với } \\
\text { đồng nghiệp }\end{array}$ & \multirow{4}{*}{$\begin{array}{l}\text { (Sakano, Obeng, \& Fuller, } \\
\text { 2016) }\end{array}$} \\
\hline 2 & $\begin{array}{l}\text { Tôi rất vui khi có cơ hội chia sẻ kiến thức với đồng } \\
\text { nghiệp }\end{array}$ & \\
\hline 3 & $\begin{array}{l}\text { Tôi cho rằng việc chia sẻ kiến thức với đồng nghiệp } \\
\text { là một việc làm có ý nghĩa }\end{array}$ & \\
\hline 4 & $\begin{array}{l}\text { Tôi chia sẻ kinh nghiệm với đồng nghiệp một cách } \\
\text { nhiệt tình }\end{array}$ & \\
\hline \multicolumn{3}{|c|}{ Vốn xã hội của tổ chức (OSC) } \\
\hline 5 & Các nhân viên cùng hướng đến tầm nhìn của đơn vị & \multirow{8}{*}{$\begin{array}{c}\text { (Kianto \& Waajakoski, } \\
\text { 2010; Kim, 2018; Leana \& } \\
\text { Pil, 2006) }\end{array}$} \\
\hline 6 & $\begin{array}{l}\text { Các nhân viên cùng theo đuổi mục tiêu và sứ mệnh } \\
\text { tập thể }\end{array}$ & \\
\hline 7 & Các nhân viên có cùng mục đích trong công việc & \\
\hline 8 & Các nhân viên cùng thực hiện kế hoạch của đơn vị & \\
\hline 9 & Các nhân viên thân ái giúp đỡ lẫn nhau & \\
\hline 10 & Các nhân viên tin tưởng nhau trong công việc & \\
\hline 11 & Các nhân viên trung thực trong công việc & \\
\hline 12 & Các nhân viên đề cao tinh thần tập thể & \\
\hline
\end{tabular}




\begin{tabular}{|c|c|c|}
\hline & Thang đo & Nguồn tham khảo \\
\hline \multicolumn{3}{|c|}{ Động lực phụng sự (PSM) } \\
\hline 13 & $\begin{array}{l}\text { Tôi quan tâm đến những lợi ích mà công việc của } \\
\text { tôi mang đến cộng đồng }\end{array}$ & \multirow{6}{*}{$\begin{array}{c}\text { (Wright et al., 2016) và thảo } \\
\text { luận chuyên gia }\end{array}$} \\
\hline 14 & $\begin{array}{l}\text { Tôi có động lực làm việc tốt hơn vì tôi muốn giúp } \\
\text { đỡ người khác }\end{array}$ & \\
\hline 15 & $\begin{array}{l}\text { Tôi sẵn sàng hi sinh lợi ích cá nhân vì lợi ích của } \\
\text { đơn vị }\end{array}$ & \\
\hline 16 & $\begin{array}{l}\text { Cơ hội thăng tiến thúc đẩy tôi thực hiện tốt công } \\
\text { việc của mình }\end{array}$ & \\
\hline 17 & $\begin{array}{l}\text { Tôi sẽ không hài lòng nếu trong vòng } 03 \text { đến } 05 \\
\text { năm tới không có sự thăng tiến trong công việc }\end{array}$ & \\
\hline 18 & $\begin{array}{l}\text { Tôi sẽ hài lòng hơn với công việc nếu được đề bạt } \\
\text { lên vị trí cao hơn }\end{array}$ & \\
\hline \multicolumn{3}{|c|}{ Chia sẻ kiến thức (KS) } \\
\hline 19 & $\begin{array}{l}\text { Tôi chủ động chia sẻ kiến thức liên quan đến công } \\
\text { việc của mình cho đồng nghiệp }\end{array}$ & \multirow{6}{*}{ (Kim, 2018) } \\
\hline 20 & $\begin{array}{l}\text { Tôi chia sẻ với đồng nghiệp những kỹ năng và kinh } \\
\text { nghiệm làm việc hiệu quả }\end{array}$ & \\
\hline 21 & $\begin{array}{l}\text { Tôi chia sẻ cách thức giải quyết công việc khi gặp } \\
\text { vấn đề phát sinh }\end{array}$ & \\
\hline 22 & $\begin{array}{l}\text { Khi thấy kiến thức mới có ích cho công việc, tôi } \\
\text { khuyến khích mọi người học nó }\end{array}$ & \\
\hline 23 & $\begin{array}{l}\text { Tôi chia sẻ kiến thức của mình với nhiều người } \\
\text { trong đơn vị }\end{array}$ & \\
\hline 24 & Tôi chủ động trả lời những thắc mắc của đồng nghiệp & \\
\hline
\end{tabular}

Nguồn: Tác giả tổng hợp

Phạm vi nghiên cứu: tác giả thực hiện việc khảo sát theo phương pháp thuận tiện. Phiếu điều tra khảo sát được thu thập từ các học viên tham gia các lớp học chính trị tại Học viện Cán bộ Thành phố Hồ Chí Minh. Các học viên này đang công tác từ nhiều ngành nghề khác nhau thuộc khu vực công như được trình bày trong Bảng 2 bên dưới.

Sau khi tiến hành khảo sát các tác giả sử dụng SPSS 22 và AMOS 22 để phân tích thống kê. Đầu tiên, phân tích nhân tố khám phá (EFA) được áp dụng toàn bộ các thang đo để đánh giá sơ bộ về khuynh hướng tương quan nhóm các biến quan sát, giá trị hội tụ và giá trị phân biệt. Thứ hai, phân tích yếu tố khẳng định (CFA) được tiến hành để kiểm tra toàn bộ mô hình được xây dựng, bao gồm bốn khái niệm và các biến quan sát tương ứng.

\section{Kết quả}

\subsection{Thống kê mô tả}

Các tác giả đã nhận được sự hỗ trợ quý giá từ Học viện Cán bộ Thành phố Hồ Chí Minh. Đây là đơn vị tổ chức toàn bộ lớp học chính trị cho các lãnh đạo đương nhiệm đang công tác tại các đơn vị hành chính sự nghiệp và các tổ chức xã hội tại Thành phố Hồ Chí Minh. Cụ thể, phiếu sát được thu thập tại 20 lớp chính trị trung cấp. Mất bốn tháng, từ tháng 11 năm 2018 đến tháng 
02 năm 2019 để liên lạc và phát 350 bảng hỏi tại các lớp chính trị cho các lãnh đạo đương nhiệm.

Trong 350 mẫu được phát ra và thu về có 22 phiếu đánh hoàn toàn rất đồng ý hoặc hoàn toàn không đồng ý ở tất cả các câu, những phiếu này bị loại do sẽ làm thiên lệch đến kết quả nghiên cứu, cùng đó có 09 phiếu khảo sát còn có nhiều câu không trả lời đầy đủ vì vậy tác giả đã loại bỏ hoàn toàn 31 mẫu đó để tránh ảnh hưởng đến kết quả của nghiên cứu. Cuối cùng, các tác giả nhận được 319 bảng trả lời hợp lệ. Bảng 2 cung cấp thông tin chi tiết về nhân khẩu học của người trả lời và thông tin tổ chức nơi họ làm việc.

\section{Bảng 2}

Thống kê mô tả thông tin người trả lời phỏng vấn

\begin{tabular}{|lcc|}
\hline \multicolumn{1}{|c|}{ Đặc tính } & Số lưộng $(\mathbf{N}=\mathbf{3 1 9})$ & Tỷ lệ \% \\
\hline Giới tính & 150 & \\
Nam & 169 & $47.02 \%$ \\
Nữ & & $52.98 \%$ \\
\hline Độ tuổi & 27 & \\
18 to 25 & 138 & $8.46 \%$ \\
26 to 35 & 120 & $43.26 \%$ \\
36 to 45 & 31 & $37.62 \%$ \\
46 to 55 & 3 & $9.72 \%$ \\
Over 55 & & $0.90 \%$ \\
\hline Trình độ học vấn & 244 & $76.48 \%$ \\
Trình độ đại học & 71 & $22.25 \%$ \\
Trình độ thạc sĩ & 4 & $1.27 \%$ \\
Khác & & \\
\hline Đang công tác tại & 85 & $26.64 \%$ \\
Trường công lập & 69 & $21.63 \%$ \\
Doanh nghiệp có vốn nhà nước & 42 & $13.17 \%$ \\
Bệnh viện, phòng khám và trạm y tế & 123 & $38.56 \%$ \\
Ũy ban nhân nhân quận huyện và phường xã & & \\
\hline
\end{tabular}

Nguồn: Tác giả tổng hợp

\subsection{Kết quả đánh giá độ tin cậy, giá trị hội tụ, giá trị phân biệt}

Bảng 3 cho thấy bảng hỏi tự điền và thống kê mô tả của việc thu thập dữ liệu, bao gồm giá trị trung bình và độ lệch chuẩn. Cụ thể, về độ tin cậy, hệ số Cronbach's Alpha của tất cả biến đều lớn hơn 0.8 . Hơn nữa, kết quả thử nghiệm cũng cho thấy tương quan biến tổng của tất cả biến quan sát đều lớn hơn 0.5 , đáp ứng được độ tin cậy của yêu cầu thang đo (Darren \& Mallery, 2003). Hệ số Cronbach's Alpha lần lượt của thang đo thái độ, vốn xã hội của tổ chức, động lực phụng sự và chia sẻ kiến thức là $0.887 ; 0.897 ; 0.811$ và 0.911 .

Kết quả phân tích nhân tố khám phá (EFA), ta có kết quả thể hiện Bảng 4 như sau: biến quan sát OSC4 bị loại vì kết quả nhân tố tải nhỏ hơn 0.5 . Bên cạnh đó biến vốn xã hội của tổ chức và động lực phụng sự bị tách ra làm hai nhân tố phụ. Trong đó, vốn tổ chức xã hội được 
tách biệt khỏi hai tiểu nhân tố là khả năng gắn kết cộng đồng (OSC A) và xây dựng niềm tin trong cộng đồng (OSC_B); kế đến biến động lực phụng sự cũng được tách làm hai nhân tố phụ gồm động lực phục sự vì tập thể (PSM_A) và động lực phục sự vì phát triển sự nghiệp (PSM_B).

\section{Bảng 3}

Kết quả phân tích hệ số Cronbach's Alpha

\begin{tabular}{|c|c|c|c|c|}
\hline Construct & $\begin{array}{c}\text { Items } \\
\text { (variable } \\
\text { coding) }\end{array}$ & Mean & SD & Alpha \\
\hline \multicolumn{2}{|l|}{ Thái độ (ATT) } & & & $\mathbf{0 . 8 8 7}$ \\
\hline $\begin{array}{l}\text { Tôi chia sẻ kiến thức công việc một cách vui vẻ với } \\
\text { đồng nghiệp }\end{array}$ & (ATT1) & 4.45 & 0.69 & \\
\hline $\begin{array}{l}\text { Tôi rất vui khi có cơ hội chia sẻ kiến thức với đồng } \\
\text { nghiệp }\end{array}$ & $(\mathrm{ATT} 2)$ & 4.41 & 0.68 & \\
\hline $\begin{array}{l}\text { Tôi cho rằng việc chia sẻ kiến thức với đồng nghiệp là } \\
\text { một việc làm có ý nghĩa }\end{array}$ & (ATT3) & 4.45 & 0.67 & \\
\hline $\begin{array}{l}\text { Tôi chia sẻ kinh nghiệm với đồng nghiệp một cách } \\
\text { nhiệt tình }\end{array}$ & (ATT4) & 4.32 & 0.73 & \\
\hline \multicolumn{2}{|l|}{ Vốn xã hội của tổ chức (OSC) } & & & 0.897 \\
\hline Các nhân viên cùng hướng đến tầm nhìn của đơn vị & (OSC1) & 3.81 & 0.85 & \\
\hline $\begin{array}{l}\text { Các nhân viên cùng theo đuổi mục tiêu và sứ mệnh tập } \\
\text { thể }\end{array}$ & $(\mathrm{OSC} 2)$ & 3.72 & 0.84 & \\
\hline Các nhân viên có cùng mục đích trong công việc & (OSC3) & 3.71 & 0.81 & \\
\hline Các nhân viên cùng thực hiện kế hoạch của đơn vị & $(\mathrm{OSC} 4)$ & 4.00 & 0.69 & \\
\hline Các nhân viên thân ái giúp đỡ lẫn nhau & (OSC5) & 3.84 & 0.87 & \\
\hline Các nhân viên tin tưởng nhau trong công việc & (OSC6) & 3.62 & 0.89 & \\
\hline Các nhân viên trung thực trong công việc & (OSC7) & 3.59 & 0.91 & \\
\hline Các nhân viên đề cao tinh thần tập thể & $(\mathrm{OSC} 8)$ & 3.72 & 0.86 & \\
\hline \multicolumn{2}{|l|}{ Động lực phụng sự (PSM) } & & & 0.811 \\
\hline $\begin{array}{l}\text { Tôi quan tâm đến những lợi ích mà công việc của tôi } \\
\text { mang đến cộng đồng }\end{array}$ & (PSM1) & 4.02 & 0.94 & \\
\hline $\begin{array}{l}\text { Tôi có động lực làm việc tốt hơn vì tôi muốn giúp đỡ } \\
\text { người khác }\end{array}$ & (PSM2) & 3.95 & 0.92 & \\
\hline Tôi sẵn sàng hi sinh lợi ích cá nhân vì lợi ích của đơn vị & (PSM3) & 3.71 & 0.90 & \\
\hline $\begin{array}{l}\text { Cơ hội thăng tiến thúc đẩy tôi thực hiện tốt công việc } \\
\text { của mình }\end{array}$ & (PSM4) & 3.60 & 1.02 & \\
\hline $\begin{array}{l}\text { Tôi sẽ không hài lòng nếu trong vòng } 3 \text { đến } 5 \text { năm tới } \\
\text { không có sự thăng tiển trong công việc }\end{array}$ & $(\mathrm{PSM} 5)$ & 3.37 & 1.05 & \\
\hline $\begin{array}{l}\text { Tôi sẽ hài lòng hơn với công việc nếu được đề bạt lên vị } \\
\text { trí cao hơn }\end{array}$ & $(\mathrm{PSM})$ & 3.39 & 1.06 & \\
\hline
\end{tabular}




\begin{tabular}{|c|c|c|c|c|}
\hline Construct & $\begin{array}{c}\text { Items } \\
\text { (variable } \\
\text { coding) }\end{array}$ & Mean & SD & Alpha \\
\hline \multicolumn{2}{|l|}{ Chia sẻ kiến thức (KS) } & & & 0.911 \\
\hline $\begin{array}{l}\text { Tôi chủ động chia sẻ kiến thức liên quan đến công việc } \\
\text { của mình cho đồng nghiệp }\end{array}$ & $(\mathrm{KS} 1)$ & 4.06 & 0.84 & \\
\hline $\begin{array}{l}\text { Tôi chia sẻ với đồng nghiệp những kỹ năng và kinh } \\
\text { nghiệm làm việc hiệu quả }\end{array}$ & $(\mathrm{KS} 2)$ & 4.16 & 0.74 & \\
\hline $\begin{array}{l}\text { Tôi chia sẻ cách thức giải quyết công việc khi gặp vấn } \\
\text { đề phát sinh }\end{array}$ & $(\mathrm{KS} 3)$ & 4.14 & 0.69 & \\
\hline $\begin{array}{l}\text { Khi thấy kiến thức mới có ích cho công việc, tôi khuyến } \\
\text { khích mọi người học nó }\end{array}$ & $(\mathrm{KS} 4)$ & 4.16 & 0.72 & \\
\hline $\begin{array}{l}\text { Tôi chia sẻ kiến thức của mình với nhiều người trong } \\
\text { đơn vị }\end{array}$ & (KS5) & 3.96 & 0.81 & \\
\hline Tôi chủ động trả lời những thắc mắc của đồng nghiệp & (KS6) & 4.03 & 0.79 & \\
\hline
\end{tabular}

Ghi chú: SD: Độ lệch chuẩn; sử dụng thang đo 5 mức độ: 1 là rất không đồng ý; 5 là hoàn toàn đồng ý Nguồn: Tác giả tự tổng hợp

\section{Bảng 4}

Ma trận xoay

\begin{tabular}{|c|c|c|c|c|c|c|}
\hline & \multicolumn{6}{|c|}{ Factor } \\
\hline & 1 & 2 & 3 & 4 & 5 & 6 \\
\hline ATT1 & & & .862 & & & \\
\hline ATT2 & & & .889 & & & \\
\hline ATT3 & & & .700 & & & \\
\hline ATT4 & & & .708 & & & \\
\hline OSC1 & & & & & & .830 \\
\hline OSC2 & & & & & & .988 \\
\hline OSC3 & & & & & & .514 \\
\hline OSC5 & & .834 & & & & \\
\hline OSC6 & & .874 & & & & \\
\hline OSC7 & & .904 & & & & \\
\hline OSC 8 & & .704 & & & & \\
\hline PSM1 & & & & .900 & & \\
\hline PSM2 & & & & .903 & & \\
\hline PSM3 & & & & .671 & & \\
\hline PSM4 & & & & & .718 & \\
\hline PSM5 & & & & & .895 & \\
\hline PSM6 & & & & & .862 & \\
\hline KS1 & .888 & & & & & \\
\hline KS2 & .900 & & & & & \\
\hline KS3 & .886 & & & & & \\
\hline KS4 & .678 & & & & & \\
\hline KS5 & .790 & & & & & \\
\hline KS6 & .578 & & & & & \\
\hline
\end{tabular}

Nguồn: Trích kết quả phân tích số liệu từ phần mềm SPSS 
Kết quả kiểm nghiệm CFA cho toàn bộ mô hình cho thấy số liệu thống kê sự phù hợp của mô hình hồi quy và chỉ ra hệ số phù hợp mô hình tốt $(\mathrm{CMIN} / \mathrm{df}=1.894, \mathrm{CFI}=0.959, \mathrm{SRMS}=$ 0.056, RMSEA = 0.053. Vì vây, toàn bộ chỉ số phù hợp mô hình đáp ứng được yêu cầu.

Để kiểm định giá trị hội tụ của thang đo, hai tiêu chí phải được cân nhắc là phương sai trích (AVE) và độ tin cậy tổng hợp $(\mathrm{CR})$. Giá trị tối thiểu của AVE phải lớn hơn 0.5 , và giá trị tối thiểu của CR lớn hơn 0.6 để đảm bảo giá trị hội tụ khái niệm (Fornell \& Larcker, 1981). Sau khi tiến hành CFA, tính hợp lệ hội tụ đã đạt chuẩn khi các giá trị đo lường $C R$ và $A V E$ của tất cả các biến đều lần lượt lớn hơn 0.7 và 0.5 . Vì vậy, tính hợp lệ hội tụ cũng như độ tin cậy từ tất cả các yếu tố đạt yêu cầu.

\section{Bảng 5}

Độ tin cậy toàn diện của cấu trúc bậc hai

\begin{tabular}{|l|c|c|c|c|c|c|c|c|c|c|}
\hline & CR & AVE & MSV & MaxR(H) & OSC_B & KS & OSC_A & ATT & PSM_A & PSM_B \\
\hline OSC_B & 0.863 & 0.681 & 0.360 & 0.898 & $\mathbf{0 . 8 2 6}$ & & & & & \\
\hline KS & 0.912 & 0.636 & 0.450 & 0.927 & 0.404 & $\mathbf{0 . 7 9 7}$ & & & & \\
\hline OSC_A & 0.897 & 0.685 & 0.360 & 0.903 & 0.600 & 0.387 & $\mathbf{0 . 8 2 8}$ & & & \\
\hline ATT & 0.887 & 0.663 & 0.450 & 0.893 & 0.366 & 0.671 & 0.373 & $\mathbf{0 . 8 1 4}$ & & \\
\hline PSM_A & 0.876 & 0.702 & 0.257 & 0.894 & 0.307 & 0.399 & 0.388 & 0.507 & $\mathbf{0 . 8 3 8}$ & \\
\hline PSM_B & 0.866 & 0.682 & 0.101 & 0.869 & 0.149 & 0.098 & 0.167 & 0.170 & 0.318 & $\mathbf{0 . 8 2 6}$ \\
\hline
\end{tabular}

Nguồn: Kết quả phân tích số liệu

Hình 2 mô tả những phát hiện từ kết quả kiểm định các giả thuyết theo phương pháp SEM. Số liệu thống kê của sự phù hợp của mô hình hồi quy tổng thể được chấp nhận. Dữ liệu có được củng cố Giả thuyết 01 - vốn xã hội của tổ chức có ảnh hưởng đáng kể đến việc chia sẻ kiến thức $(0.271, \mathrm{p}<0.05)$. Kế đó, các phát hiện cũng cho thấy vốn xã hội của tổ chức có liên hệ tích cực đến thái độ (ước lượng $=0.567, \mathrm{p}<0.001$ ). Do đó, Giả thuyết 02 được củng cố.

Mối quan hệ thái độ tác động lên chia sẻ kiến thức đã được kiểm tra là tích cực với ước lượng $=0.519$ và $\mathrm{p}<0.001$. Do vậy, Giả thuyết 03 được củng cố. Ngoài ra, Giả thuyết 04 đúng vì hệ số tương quan được ước lượng xác nhận mối liên hệ tích cực và đáng kể về mặt thống kê giữa vốn xã hội của tổ chức và động lực phụng sự $(0.614, \mathrm{p}<0.001)$. Tuy nhiên, dữ liệu có được không cung cấp bằng chứng cho thấy củng cố Giả thuyết 05 . Hay nói cách khác, Giả thuyết 05 không được chấp nhận. Tổng kết về kết quả của giả thuyết, tác giả đã thiết lập mô hình kết quả như dưới đây.

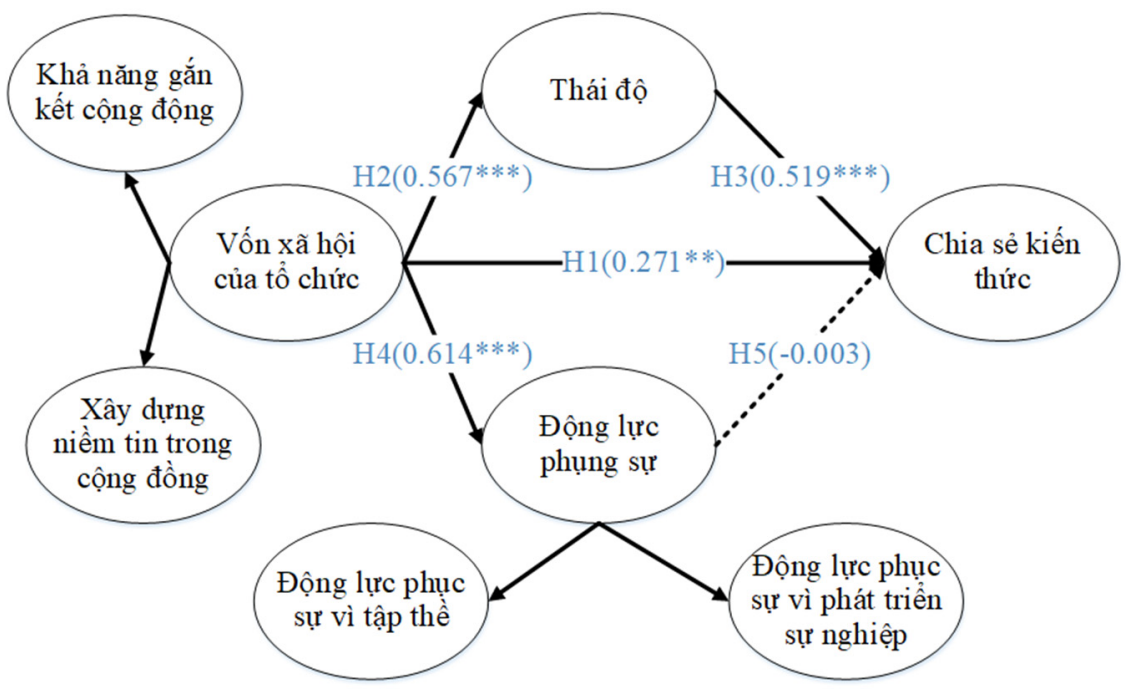

Hình 2. Kết quả kiểm định giả thuyết $(* * * \mathrm{p}<0.001 ; * * \mathrm{p}<0.05)$ 


\section{Thảo luận và kiến nghị}

\subsection{Tác động tích cục của thái độ và vốn tổ chức của xã hội}

Trong bối cảnh thời đại số, chia sẻ kiến thức đã phát triển từ một khái niệm mới nổi thành một hoạt động ngày càng phổ biến ở các tổ chức thuộc khu vực công. Nghiên cứu này phát hiện ra mối hệ giữa thái độ, động lực phụng sự, vốn xã hội của tổ chức với việc chia sẻ kiến thức tại khu vực công ở Việt Nam. Kết quả chỉ ra rằng thái độ, vốn xã hội của tổ chức có mối quan hệ tích cực với chia sẻ kiến thức, tương tự như kết quả của các nghiên cứu trước đây (Bock et al., 2005; Chow \& Chan, 2008). Đặc biệt, thái độ của nhân viên đối với chia sẻ kiến thức cơ bản được thúc đẩy bởi các mối quan hệ hỗ trợ liên quan đến chia sẻ kiến thức. Nó cũng chỉ ra rằng lãnh đạo ở các tổ chức thuộc khu vực công có thể gia tăng hành vi chia sẻ kiến thức ở cơ quan bằng cách tạo môi trường làm việc sao cho khuyến khích thái độ tích cực trong đội ngũ công viên chức, linh hoạt phân bổ nguồn lực xã hội có sẵn, tạo ra định hướng mục tiêu tập thể, chia sẻ niềm tin. Ngoài ra, công - viên chức cũng sẽ sẵn lòng chia sẻ kiến thức hơn vì họ tin tưởng lẫn nhau vận dụng kiến thức một cách cẩn thận và sử dụng kiến thức thích hợp sẽ mang lại lợi ích cho tổ chức (Choi, 2016).

Ngoài ra, các thông tin đúc kết được xác nhận mối quan hệ tích cực giữa vốn xã hội của tổ chức với thái độ (Chow \& Chan, 2008) và động lực phụng sự (Moynihan \& Pandey, 2007) và ngụ ý rằng các tổ chức công có cơ hội và trách nhiệm tạo ra môi trường mà nơi đó nhân viên cảm thấy họ đang có những đóng góp cho lợi ích công.

\subsection{Nhân tố động lục phụng sụ̣ ảnh hưởng đến chia sẻ kiến thức}

Nghiên cứu này phát triển các mô hình nghiên cứu đi trước về dự định chia sẻ kiến thức trong khu vực công (Bock et al., 2005; Chen et al., 2014; Kim, 2018). Tuy nhiên, trong bối cảnh nghiên cứu ở khu vực công tại Việt Nam, động lực phụng sự không trực tiếp đóng vai trò thiết yếu trong việc khuyến khích quá trình chia sẻ kiến thức. Phát hiện này cũng chứng tỏ rằng cần có nghiên cứu khác mở rộng các chiều thông tin cá nhân về khái niệm động lực phụng sự (Perry \& Vandenabeele, 2015). Quả thật, thang đo động lực phụng sự trong nghiên cứu này được kết hợp từ ba biến quan sát áp dụng từ (Wright et al., 2016) và ba biến quan sát tự hình thành từ kết quả của bốn nhóm thảo luận. Vì thế, việc phát triển ba biến quan sát động lực phụng sự tự hình thành không dẫn đến tác động trực tiếp nào lên quá trình chia sẻ kiến thức ở khu vực công tại Việt Nam. Kết quả này ủng hộ quan điểm rằng các chiều thông tin về động lực phụng sự khác nhau có thể dẫn đến các tiền lệ khác nhau và các nền tảng lý thuyết nhất định (Kim \& Vandenabeele, 2010).

\subsection{Kiến nghị}

Mục tiêu cuối cùng của việc chia sẻ kiến thức là nâng cao hiệu suất và hiệu quả trong công việc. Từ những phát hiện trong nghiên cứu này, tác giả đề xuất một số giải pháp trong quản trị nhân sự để duy trì và tăng cường hoạt động chia sẻ kiến thức trong tổ chức. Thứ nhất, lãnh đạo ở các tổ chức công có thể gia tăng hành vi chia sẻ kiến thức ở cơ quan bằng cách tạo khuyến khích thái độ tích cực trong đội ngũ nhân viên, linh hoạt phân bổ nguồn lực xã hội có sẵn, tạo ra định hướng mục tiêu tập thể, chia sẻ lòng tin.

Bài học thứ hai là lãnh đạo của các tổ chức công đóng vai trò quan trọng trong việc tạo điều kiện cho quá trình chia sẻ kiến thức bằng cách tạo môi trường làm việc thân thiện, tăng khả năng gắn kết trong cộng đồng và xây dựng niềm tin với các thành viên trong tổ chức. Trong các tổ chức công, lãnh đạo trước hết phải tạo môi trường công sở thuận lợi để công - viên chức được khuyến khích chia sẻ quan điểm, kinh nghiệm, và kiến thức của mình: Có các buổi nói chuyện về chuyên đề giữa các phòng ban trong tổ chức. Bằng cách tạo ra những cơ hội chia sẻ kiến thức trong và ngoài giờ làm việc như tổ chức các buổi sinh hoạt cuối tuần, trong các ngày lễ tất cả các 
thành viên trong tổ chức tham gia, giao lưu để hiểu nhau hơn, tạo sự kết nối và chia sẻ. Khen thưởng các hoạt động chia sẻ kiến thức, công - viên chức có thể nhận ra được các đóng góp của họ có ý nghĩa như thế nào đối với mọi người xung quanh và dịch vụ công.

Nghiên cứu cũng có những hạn chế nhất định. Việc đo lường các nhân tố được áp dụng từ các tài liệu viết bằng tiếng Anh, và một số biến quan sát được áp dụng từ các tác giả nước ngoài có thể tạo ra một số rào cản vô hình khi bảng hỏi được dịch sang tiếng Việt. Các thông tin có được cũng cho thấy cần thêm nhiều nghiên cứu khác về mối quan hệ giữa thái độ, động lực dịch vụ công, vốn xã hội của tổ chức. Ngoài ra, cũng cần có một nghiên cứu sâu hơn tập trung phát triển mô hình nghiên cứu cho các khu vực khác như công ty và tập đoàn đa quốc gia. Việc xây dựng biến quan sát liên quan đến động lực dịch vụ công có thể được cân nhắc để làm phong phú hơn các chiều thông tin dựa trên công trình gốc của (Perry, 1996) hay công trình sau của (Kim, 2009, 2018). Các nghiên cứu về sau cần phải cân nhắc những hạn chế này và phát triển mở rộng mô hình nghiên cứu bao quát hơn.

\section{LÒ̀I CÁM ONN}

Nghiên cứu này được tài trợ bởi Trường Đại học Quốc tế, Đại học Quốc gia Thành phố Hồ Chí Minh theo mã số đề tài SV2019-CPA-04.

\section{Tài liệu tham khảo}

Adler, P. S., \& Kwon, S. W. (2002). Social capital: Prospects for a new concept. Academy of Management Review, 27(1), 17-40. doi:10.5465/AMR.2002.5922314

Ajzen, I., \& Fishbein, M. (1980). Understanding attitudes and predicting social behavior. Englewood Cliffs, NJ: Prentice-Hall.

Alavi, M., \& Leidner, D. E. (2001). Knowledge management and knowledge management systems: Conceptual foundations and research issues. MIS Quarterly, 25(1), 107-136.

Andrews, R. (2012). Social capital and public service performance: A review of the evidence. Public Policy and Administration, 27(1), 49-67. doi:10.1177/0952076710394399

Bandura, A. (1989). Human agency in social cognitive theory. American Psychologist, 49(9), 1175-1184. doi:10.1037/0003-066X.44.9.1175

Bartol, K. M., \& Srivastava, A. (2002). Encouraging knowledge sharing: The role of organizational reward systems. Journal of Leadership \& Organizational Studies, 9(1), 6476. doi:10.1177/107179190200900105

Bock, G.-W., Zmud, R., Kim, Y.-G., \& Lee, J.-N. (2005). Behavioral intention formation in knowledge sharing: Examining the roles of extrinsic motivators, social-psychological factors, and organizational climate. MIS Quarterly, 29(1), 87-111. doi:10.1016/j.knosys.2012.02.001

Boland, R. J., \& Tenkasi, R. V. (1995). Perspective making and perspective taking in communities of knowing. Organization Science, 6(4), 350-372. doi:10.1287/orsc.6.4.350

Brewer, G. A., \& Seiden, S. C. (1998). Whistle blowers in the federal civil service: New evidence of the public service ethic. Journal of Public Administration Research and Theory, 8(3), 413-440. doi:10.4324/9780429497957

Brislin, R. W. (1970). Back-translation for cross-cultural research. Journal of Cross-Cultural Psychology, 1(3), 185-216. doi:10.1177/135910457000100301 
Coleman, J. S. (1988). Social capital in the creation of human capital. American Journal of Sociology, 94, S95-S120. doi:10.1086/228943

Coursey, D. H., \& Pandey, S. K. (2007). Public service motivation measurement: Testing an abridged version of Perry's proposed scale. Administration and Society, 39(5), 547-568. doi:10.1177/0095399707303634

Chen, C. A., Hsieh, C. W., \& Chen, D. Y. (2014). Fostering public service motivation through workplace trust: Evidence from public managers in Taiwan. Public Administration, 92(4), 954-973. doi:10.1111/padm.12042

Chiu, C. M., Hsu, M. H., \& Wang, E. T. G. (2006). Understanding knowledge sharing in virtual communities: An integration of social capital and social cognitive theories. Decision Support Systems, 42(3), 1872-1888. doi:10.1016/j.dss.2006.04.001

Choi, Y. (2016). The impact of social capital on employees' knowledge-sharing behavior: An empirical analysis of U.S. federal agencies. Public Performance and Management Review, 39(2), 381-405. doi:10.1080/15309576.2015.1108795

Chow, W. S., \& Chan, L. S. (2008). Social network, social trust and shared goals in organizational knowledge sharing. Information and Management, 45(7), 458-465. doi:10.1016/j.im.2008.06.007

Darren, G., \& Mallery, P. (2003). SPSS for windows step by step: Answers to selected exercises. A simple guide and reference. Boston, MA: Allyn and Bacon.

Darvish, H., \& Nikbakhsh, R. (2010). Studying the relations of social capital factors with knowledge sharing: A case study at research department of Irib. Transylvanian Review of Administrative Sciences, 31E, 28-47.

Fornell, C., \& Larcker, D. F. (1981). Evaluating structural equation models with unobservable variables and measurements error. Journal of Marketing Research, 18(4), 39-50. doi: $10.2307 / 3151312$

Gagne, R. M., \& Medsker, K. L. (1996). The conditions of learning: Training applications. Fort Worth, TE: Harcourt Brace College Publishers.

Hsu, M. H., Ju, T. L., Yen, C. H., \& Chang, C. M. (2007). Knowledge sharing behavior in virtual communities: The relationship between trust, self-efficacy, and outcome expectations. International Journal of Human Computer Studies, 65(2), 153-169. doi:10.1016/j.ijhcs.2006.09.003

Huang, M.-H., \& Chang, S.-H. (2017). Similarities and differences in East Asian confucian culture: A comparative analysis. OMNES: The Journal of Multicultural Society, 7(2), 1-40. doi:10.15685/omnes.2017.01.7.2.1

Jackson, S. E., Chuang, C. H., Harden, E. E., \& Jiang, Y. (2006). Toward developing human resource management systems for knowledge-intensive teamwork. Research in Personnel and Human Resources Management, 25, 27-70. doi:10.1016/S0742-7301(06)25002-3

Jacobs, J. (1961). The death and life of great American cities. New York, NY: Random House.

Kianto, A., \& Waajakoski, J. (2010). Linking social capital to organizational growth. Knowledge Management Research and Practice, 8(1), 4-14. doi:10.1057/kmrp.2009.29

Kim, S. (2009). Revising Perry's measurement scale of public service motivation. American Review of Public Administration, 39(2), 149-163. doi:10.1177/0275074008317681 
Kim, S. (2018). Public service motivation, organizational social capital, and knowledge sharing in the Korean public sector. Public Performance and Management Review, 41(1), 130-151. doi:10.1080/15309576.2017.1358188

Kim, S., \& Vandenabeele, W. (2010). A strategy for building public service motivation research internationally. Public Administration Review, 70(5), 701-709. doi:10.1111/j.15406210.2010.02198.x

Leana, C. R., \& Pil, F. K. (2006). Social capital and organizational performance: Evidence from urban public schools. Organization Science, 17(3), 353-366. doi:10.1287/orsc.1060.0191

Leana, C. R., \& Buren, H. J. V. (1999). Organizational social capital and employment practices. The Academy of Management Review, 24(3), 538-555.

Lee, D. J., \& Ahn, J. H. (2007). Reward systems for intra-organizational knowledge sharing. European Journal of Operational Research, 180(2), 938-956. doi:10.1016/j.ejor.2006.03.052

Lin, Q., Ye, D., \& Bi, B. (2014). A longitudinal and multilevel investigation on factors influencing knowledge sharing behavior. Journal of Management Policy \& Practice, 15(3), 88-101.

Martinez, M. G. (2017). Inspiring crowdsourcing communities to create novel solutions: Competition design and the mediating role of trust. Technological Forecasting and Social Change, 117, 296-304. doi:10.1016/j.techfore.2016.11.015

Moynihan, D. P., \& Pandey, S. K. (2007). The role of organizations in fostering public service motivation. Public Administration Review, 67(1), 40-53.

Pan, S. L., \& Scarbrough, H. (1998). A socio-technical view of knowledge-sharing at Buckman. Journal of Knowledge Management, 2(1), 55-66.

Perry, J. L. (1996). Measuring public service motivation: An assessment of construct reliability and validity. Journal of Public Administration Research and Theory, 6(1), 5-22.

Perry, J. L., \& Vandenabeele, W. (2015). Public service motivation research: Achievements, challenges, and future directions. Public Administration Review, 75(5), 692-699. doi:10.1111/puar.12430

Prusak, L., \& Davenport, T. (1998). Working knowledge: How organizations manage what they know. Boston, MA: Harvard Business School Press.

Sakano, R., Obeng, K., \& Fuller, K. (2016). Airport security and screening satisfaction: A case study of U.S. Journal of Air Transport Management, 55, 129-138. doi:10.1016/J.JAIRTRAMAN.2016.05.007

Smith, E. A. (2001). The role of tacit and explicit knowledge in the workplace. Journal of Knowledge Management, 5(4), 311-321. doi:10.1108/13673270110411733

Vigoda, E. (2002). Administrative agents of democracy? A structural equation modeling of the relationship between public-sector performance and citizenship involvement. Journal of Public Administration Research and Theory, 12(2), 241-272. doi:10.1093/oxfordjournals.jpart.a003531

Vij, S., \& Farooq, R. (2014a). Knowledge sharing orientation and its relationship with business performance: A structural equation modeling approach. IUP Journal of Knowledge Management, 12(3), 17-41. doi:10.4018/ijkm.2014070103 
Vij, S., \& Farooq, R. (2014b). Multi-group moderation analysis for relationship between knowledge sharing orientation and business performance. International Journal of Knowledge Management, 10(3), 36-53. doi:10.4018/ijkm.2014070103

Wang, S., \& Noe, R. A. (2010). Knowledge sharing: A review and directions for future research. Human Resource Management Review, 20(2), 115-131. doi:10.1016/j.hrmr.2009.10.001

Wang, S., Noe, R. A., \& Wang, Z. M. (2014). Motivating knowledge sharing in knowledge management systems: A quasi-field experiment. Journal of Management, 40(4), 978-1009. doi:10.1177/0149206311412192

Wright, B. E., \& Pandey, S. K. (2008). Public service motivation and the assumption of personorganization fit: Testing the mediating effect of value congruence. Administration and Society, 40(5), 502-521. doi:10.1177/0095399708320187

Wright, B. E., Hassan, S., \& Park, J. (2016). Does a public servce ethic encourage ethical behaviour? Public service motivation, ethical leadership and the willingness to report ethical problems. Public Administration, 94(3), 647-663. doi:10.1111/padm.12248

Yang, J. T., \& Wan, C. S. (2004). Advancing organizational effectiveness and knowledge management implementation. Tourism Management, 25(5), 593-601. doi:10.1016/j.tourman.2003.08.002 